\title{
Effects of traction therapy on atlantoaxial joint dislocation-induced cervical vertigo
}

\author{
Genghui Cai ${ }^{1 \oplus}$, Dabin Zhu ${ }^{1 \oplus}$, Jieyun Chen ${ }^{2 \oplus \bowtie}$, Xiuyao Lin $^{1 \oplus \bowtie}$, and Ri Chen ${ }^{1 \oplus}$ \\ ${ }^{1}$ Department of Rehabilitation, Quanzhou First Hospital Affiliated to Fujian Medical University, Quanzhou, Fujian, China \\ ${ }^{2}$ Department of Radiology, Quanzhou First Hospital Affiliated to Fujian Medical University, Quanzhou, Fujian, China
}

\begin{abstract}
Cervical vertigo is a common complication of atlantoaxial joint dislocation. However, there is no consensus on the effects of different therapies on the recovery of the patients suffering cervical vertigo. The objective of this randomized controlled trial was to investigate the effect of traction therapy on reducing cervical vertigo induced by atlantoaxial joint dislocation. A total of 96 patients were randomized to receive traction therapy or traditional therapy for two weeks. The overall clinical efficacy was measured based on the 30-point cervical vertigo symptom and function evaluation form. The therapeutic effects were also evaluated based on lateral atlantodental space (LADS), vertigo scale, neck and shoulder pain scale, headache scale, daily life and work scale, psychosocial adaptation scale, and quality of life. Compared with the traditional therapy group, the traction group demonstrated markedly higher overall clinical efficacy $(P=0.038)$. Both the traction therapy group and the traditional therapy group showed significant decrease in LADS $(P<0.001)$, but the traction therapy group had a greater reduction of LAD compared with the traditional group $(P<0.01)$. Traction therapy consistently led to significantly greater relief of cervical vertigo symptoms, including dizziness, neck and shoulder pain, headache, inconvenience in daily living and work activities, impaired psychosocial adaptation, while improving quality of life. The efficacy of traction therapy for cervical vertigo surpasses that of traditional therapy, suggesting that traction therapy is potentially more clinically useful in treating these patients.
\end{abstract}

Key words: Atlantoaxial joint dislocation; Cervical vertigo; Traction therapy

\section{Introduction}

Atlantoaxial joint dislocation (AJD) is a common consequence of traumatic dislocation of the upper cervical spine or non-traumatic causes such as congenital causes, inflammation of the nasopharynx, and rheumatoid arthritis $(1,2)$. Therapy of AJD consists mainly of conservative manual therapy and invasive surgical therapy (3). In manual therapy, manipulation of cervical bones and muscles is performed to bring the cervical spine into the neutral position using a rotational movement, which is commonly practiced by both western chiropractors and traditional Chinese herbalists (4). However, cervical manipulation sometimes triggers a vertiginous attack that stems from decreased blood perfusion, leading to ischemic change in the cerebellum, inner ears, or brainstem (5-7). Cervical vertigo is manifested as imbalance, light-headedness, or unsteadiness. Some patients with cervical vertigo experience illusion of movement, spinning or whirling, which lead to a certain level of disability and deteriorates quality of life (8). Unfortunately, insufficient attention has been devoted to cervical vertigo in choosing the therapies for AJD. Therefore, there is an urgent need to establish effective clinical treatment methods of AJD that minimizes cervical vertigo.

The traction method for treatment of AJD is a novel technique adopted that is increasingly used in clinical practice (9-11). This method is thought to reduce cervical vertigo compared with the traditional method. The traction method has been traditionally applied to treat cervical vertigo by other spinal diseases (12-14). The benefits of traction have been shown to be an increase in blood flow (15) and a decrease in pain (16-18). However, the clinical benefit of traction therapy in alleviating cervical vertigo induced by AJD has not been fully supported due to the lack of randomized controlled clinical trials.

The aim of the study was to conduct a randomized controlled clinical trial to compare the effects of traction therapy and traditional therapy in reducing cervical vertigo, along with clinical symptoms and quality of life associated with cervical vertigo. The results provided by the study could shed light on the clinical benefit of traction therapy in reducing cervical vertigo as a treatment for AJD.

Correspondence: Jieyun Chen: <chenjieyunqz@126.com> | Xiuyao Lin: <gramer2007@163.com>

Received September 10, 2021 | Accepted January 6, 2022 


\section{Material and Methods}

\section{Enrollment}

This study was approved by the Ethic Committee of Quanzhou First Hospital Affiliated to Fujian Medical University (registration number ChiCTR2100051942). The subjects of this study were recruited from the Rehabilitation Medicine, Orthopedics, Neurology Outpatient Clinics, and wards of the First Hospital of Quanzhou City. Inclusion criteria were: 1) diagnosis of atlantoaxial disorder; 2) age between 18 and 60 years, regardless of gender; 3) dizziness caused by diseases such as cardiovascular, otolaryngology, neurology, internal medicine, orthopedics, etc.; and 4) voluntarily participation in the trial and signing the informed consent. The main symptoms used as diagnostic criteria were dizziness, migraine, neck pain, and posterior occipital pain, accompanied by symptoms of autonomic dysfunction. In severe cases, visual rotation, nausea, tinnitus, insomnia, and scalp numbness may be seen. Anatomical signs are C2 spinous process positioned to one side, swelling and pain in the tissues surrounding the vertebra, head and face skewed to the side, and limited movement of the cervical spine. These signs are aggravated when turning the head or changing position, and the neck flexion test is positive. Lateral radiographs show shallow or straightened cervical physiological curvature, normal radiographs show varying degrees of vertebral body rotation below C3, suggesting structural instability of the upper cervical segment. Open-mouth radiographs show unequal distance between atlas and teeth, with a variance of $14 \mathrm{~mm}$ and no fracture.

Exclusion criteria were: 1) patients with cervical spondylotic radiculopathy and severe spinal canal stenosis; 2) patients with history of spinal surgery or severe spine trauma; 3) known congenital variation or deformity of unstable cervical spine structure; 4) spinal infections, fractures, tumors, tuberculosis, severe spinal deformities, severe osteoporosis, ankylosing spondylitis, deformity osteitis, and other diseases seen in imaging studies; 5) severe primary diseases of the endocrine system, primary cardiovascular disease, autoimmune disease, tumor, or psychosis; 6) dizziness caused by otogenic (vestibular), ocular, cerebral (central systemic), drug-induced, poisoning, and other causes; 7) being pregnant or recently preparing for pregnancy and breastfeeding women; and 8) patients who had received or were receiving other treatments within the past 3 months, which affect the efficacy of the tested treatment.

Patients were randomized using a web-based system (Institute of Basic Research in Clinical Medicine, China Academy of Chinese Medical Science) to receive traction therapy or traditional therapy. Patients and the investigators who analyzed the data were blind to treatments.

\section{Treatment methods}

Traction therapy. The procedures for traction therapy are shown in Figure 1. The patient adopted a sitting position. To treat patients with the left deviation of the C2 spinous process, for example, the surgeon stood behind the patient and pressed the left edge of the $\mathrm{C} 2$ spinous process with the thumb of the left hand, with the right elbow flexed to clamp the patient's mandible. The surgeon placed the palm around the ears. Traction was conducted along the longitudinal axis of the spine for about $1 \mathrm{~min}$. With traction, the cervical spine was slowly rotated to the right to the $\mathrm{C} 2$ spinous process protruding position. Then a controlled, slowing increasing movement was enforced using the left thumb to push the $\mathrm{C} 2$ spinous process to the right. When a popping sound and feeling at the $\mathrm{C} 2$ spinous process was achieved, the procedure was complete.

For patients with forward incline, the patients were placed in supine position with a pillow on the neck. The surgeon sat at the top side of the patient's head, supporting both side of the patient's head using hands with the middle finger on both sides of the C2 spinous process. Cervical traction along the longitudinal axis of the spine was conducted for 2-3 $\mathrm{min}$, and then the patient was instructed to take a deep breath. At the end of exhalation, intermittent upward force was applied to the patient's head. The surgeon would look for a popping sound or a sense of movement at the $\mathrm{C} 2$ spinous process.

Traditional therapy. The steps of this therapy are shown in Figure 1. The traditional cervical spine rotation method was used. The patient was in a sitting position with the neck leaning forward by 10 to $15^{\circ}$. The physician stood behind the patient and pressed the cervical spinal

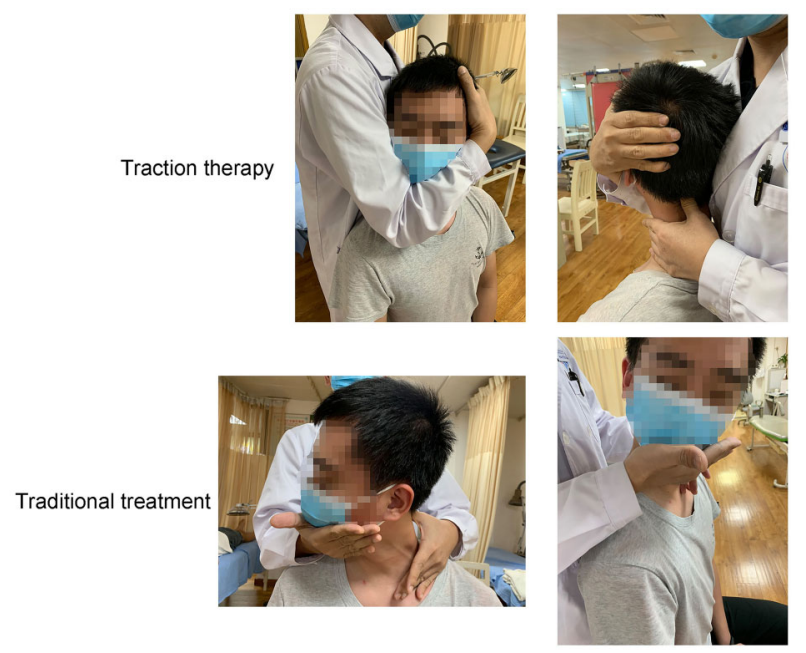

Figure 1. Procedures of traction therapy and traditional treatment. 
process or lateral process of the patient's cervical spine with one hand, while holding the chin with the other hand to slowly rotate the head. When the rotation found resistance, the physician then made a controlled larger rotation with greater force. At the same time, the spinous process was pushed hard to the opposite side with coordinated movement by the two hands. When a popping sound and feeling was achieved at the $\mathrm{C} 2$ spinous process, the manipulation was considered successful.

All patients in the two groups were treated by the same physician. According to the patient's symptoms, manipulations were performed 3 to 4 times a week. Other treatments were mainly muscle message therapies. Each treatment lasted about 15-20 min, and each treatment course was 14 days. The therapeutic effect was observed after a treatment course.

\section{Evaluation of treatment efficacy}

The primary outcomes of our trial were changes in the lateral atlanto-distal block space (LADS), cervical vertigo symptoms, and function. The secondary outcome was the quality of life of patients.

\section{Clinical efficacy}

Clinical efficacy was evaluated based on improvement of clinical symptoms and categorized as recovered, very effective, effective, and ineffective, with the following definitions: recovered: clinical symptoms disappeared and improvement rate was $\geqslant 90 \%$; very effective: clinical symptoms and signs disappeared or significantly reduced and improvement rate was $\geqslant 75 \%$; effective: clinical symptoms and signs reduced and improvement rate was $\geqslant 30 \%$; ineffective: improvement rate was $<30 \%$. Improvement rate is defined as (post-treatment points - pretreatment points) / (full score - pre-treatment points) $\times$ $100 \%$.

\section{Vertigo symptoms and patient performance}

The Neck Vertigo Symptom and Function Evaluation Scale by Chuhuai et al. (19) was used to evaluate vertigo symptoms and patient performance. The scale evaluates the degree of vertigo (8 points), vertigo frequency (4 points), duration (4 points), degree of neck and shoulder pain (4 points), headache ( 2 points), daily life and work ability (4 points), and psychological and social adaptability (4 points). Lower scores indicate less severe symptoms.

\section{Assessment of quality of life}

The MOS-SF36 scale (20) was used to assess quality of life. The scale consists of dimensions that evaluate 1) physical health; 2) social function; 3) physical role function; 4) physical pain; 5) mental health; 6) emotional role function; 7) energy; and 8) overall health. A high score indicates better quality of life.

The patients in both groups were assessed on the first day after enrollment and after the 14th day of treatment.
After six months of treatment, patients were evaluated again for the cervical vertigo symptoms, function evaluation, and MOS-SF36 scale. The number of vertigo attacks was recorded for six months.

\section{Statistical analysis}

SPSS19.0 software (IBM, USA) was used for statistical analysis. Data are reported as means $\pm S D$. The sample size was determined using PS software (Power and Sample Size Calculation version 3.0.12, <https:// biostat.app.vumc.org/wiki/Main/PowerSampleSize $>$ ). In brief, we used $0.8 \mathrm{~mm}$ as the mean value of the patients' LADS based on prior literature, while considering LADs $<0.5$ as successful recovery. Using the standard deviation of 0.51 , effect size of 0.8 , and $P<0.05$ for statistical significance, the minimal sample size was calculated to be 36. Data from before and after treatment was compared with the paired $t$-test, and between-group data was compared with the unpaired $t$-test. The chi-squared test was used to analyze categorical data.

\section{Results}

\section{Patient enrollment and characteristics}

A total of 119 patients enrolled in the study, among which 96 were included for randomization: 48 in traction therapy group and 48 in traditional therapy group. Treatment lasted 2 weeks, and post-treatment analysis was performed based on 42 patients from the traction group and 40 patients from the tradition group, due to reasons such as loss to follow-up, discontinued intervention, or unable to contact (Figure 2). The patient characteristics of the groups are summarized in Table 1. None of the characteristics were significantly different between groups.

\section{Traction therapy had higher clinical efficacy than traditional therapy}

After one course of treatment (14 days, 3-4 times a week), clinical efficacy was evaluated based on disappearance of clinical symptoms and improvement rate. Our results indicated that compared to the tradition group, a larger portion of patients in the traction group recovered (30.9 vs $22.5 \%$ ) and the treatment was deemed more effective (42.9 vs 27.5\%). Based on these data, a significantly higher clinical efficacy was found for traction therapy $(\mathrm{P}=0.038)$ (Table 2$)$.

\section{Traction therapy led to a significantly greater reduction in LADS}

Consistent with the higher clinical efficacy of traction therapy, a greater reduction of LADS was also observed in patients who received traction therapy, compared to patients who received traditional therapy $(P<0.01)$, although both therapies significantly reduced LADS compared to pre-treatment LADS $(P<0.001)$ (Figure 3). 


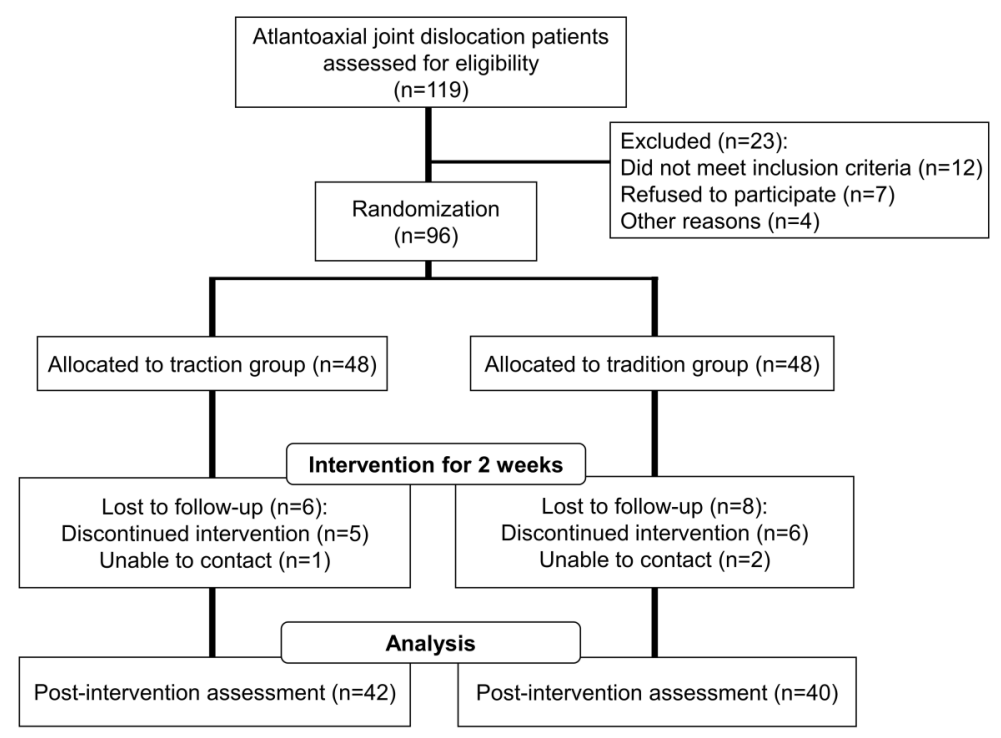

Figure 2. Flowchart of this study.

Table 1. Demographic characteristics of the included patients.

\begin{tabular}{|c|c|c|c|}
\hline \multirow[t]{2}{*}{ Variable } & \multicolumn{2}{|c|}{ Study group } & \multirow[t]{2}{*}{$\mathrm{P}$} \\
\hline & Traction group $(n=42)$ & Tradition group $(n=40)$ & \\
\hline Age (years) & $39.4 \pm 10.5$ & $41.6 \pm 11.2$ & 0.164 \\
\hline Male gender, $\mathrm{n}(\%)$ & $18(42.9 \%)$ & $22(55 \%)$ & 0.377 \\
\hline BMI & $29.4 \pm 7.2$ & $27.9 \pm 8.6$ & 0.181 \\
\hline Pre-intervention duration of disease, months & $3.65 \pm 1.78$ & $3.18 \pm 1.91$ & 0.317 \\
\hline Smokers, n (\%) & $11(26.2 \%)$ & $13(32.5 \%)$ & 0.629 \\
\hline LADS, pre-intervention (mm) & $0.92 \pm 0.18$ & $0.88 \pm 0.19$ & 0.362 \\
\hline \multicolumn{4}{|c|}{ Cervical vertigo symptom and function evaluation, pre-intervention } \\
\hline Vertigo & $8.87 \pm 2.42$ & $8.41 \pm 2.31$ & 0.469 \\
\hline Neck and shoulder pain & $2.85 \pm 0.94$ & $3.12 \pm 0.84$ & 0.275 \\
\hline Headache & $1.73 \pm 0.45$ & $1.64 \pm 0.51$ & 0.331 \\
\hline Activities of daily living and work scale & $1.94 \pm 0.68$ & $1.78 \pm 0.59$ & 0.388 \\
\hline Psychosocial adaptation & $3.33 \pm 0.86$ & $3.47 \pm 0.95$ & 0.294 \\
\hline \multicolumn{4}{|l|}{ Education } \\
\hline Junior high school and below & $16(38.1 \%)$ & $14(35.0 \%)$ & 0.788 \\
\hline Senior high school or polytechnic school & $20(47.6 \%)$ & $18(45.0 \%)$ & \\
\hline College and above & $6(14.3 \%)$ & $8(20.0 \%)$ & \\
\hline
\end{tabular}

Data are reported as means \pm SD or $\mathrm{n}(\%)$. $\mathrm{P}$ values for each group were derived from either unpaired $t$-test or Mann-Whitney test, as appropriate. Chi-squared test or Fisher's exact test was used for assessing distribution of observations or phenomena between groups. LADS: lateral atlantodental space; BMI: body mass index.

\section{Traction therapy had higher efficacy in alleviating vertigo symptoms and improving psychosocial function}

As shown in Figure 4, both therapies showed significant alleviation of vertigo symptoms, including vertigo (Figure 4A), neck and shoulder pain (Figure 4B), and headache (Figure 4C). Moreover, both therapies led to an increase in psychological function, reduction of impairment in activities of daily living and work activities (Figure 4D), and fewer psychosocial symptoms (Figure 4E). However, the effects of traction therapy were much stronger $(\mathrm{P}<0.05$ in all variables).

\section{Traction therapy was more effective in improving quality of life}

Overall, the traction group had greater improvement in physical function $(P=0.024)$, role function $(P=0.182)$, 
Table 2. Comparison of overall clinical efficacy before and after intervention.

\begin{tabular}{lcr}
\hline & \multicolumn{2}{c}{ Study group } \\
\cline { 2 - 3 } & Traction group $(\mathrm{n}=42)$ & Tradition group $(\mathrm{n}=40)$ \\
\hline Recovered & $13(30.9 \%)$ & $6(22.5 \%)$ \\
Very effective & $18(42.9 \%)$ & $12(27.5 \%)$ \\
Effective & $9(21.4 \%)$ & $14(30.0 \%)$ \\
Ineffective & $2(4.8 \%)$ & $8(20.0 \%)$ \\
$P$ & & $\mathbf{0 . 0 3 8}$ \\
\hline
\end{tabular}

Data are reported as $\mathrm{n}(\%)$. $\mathrm{P}$ values were derived from chi-squared test for assessing distribution of observations or phenomena between groups. Bold type indicates statistically significant.

physical pain $(P=0.029)$, general health perception $(P=0.031)$, and social function $(P=0.027)$ than the tradition group, which only showed significant improvement of

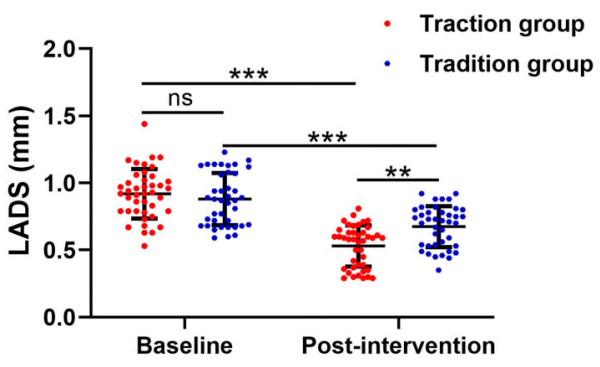

Figure 3. Effects of traction therapy on lateral atlantodental space (LADS) of patients with cervical vertigo due to atlantoaxial joint dislocation. Data are reported as means \pm SD and show all data points. ${ }^{* *} \mathrm{P}<0.01,{ }^{* * *} \mathrm{P}<0.001$, paired $t$-test and unpaired $t$-test. ns: not significant. physical function $(\mathrm{P}=0.039)$ and physical pain $(\mathrm{P}=0.041)$. (Table 3).

\section{Discussion}

AJD is an important cause of cervical vertigo (21). The vertigo caused by AJD is more common in middle-aged and elderly people, but the age of onset has decreased gradually due to the increased usage of electronic devices and computers. The incidence rate is also increasing each year (3). Furthermore, treatment of atlantoaxial joint disorder can sometimes aggravate cervical vertigo, making the choice of treatment significantly important. Cervical vertigo can seriously affect activities of daily living and work, reduce quality of life, and cause great pain to patients. At present, many clinical studies are focused on the alleviation of anatomical changes rather than vertigo and quality of life. Evidence on which treatment strategies perform better in terms of reducing vertigo are lacking.
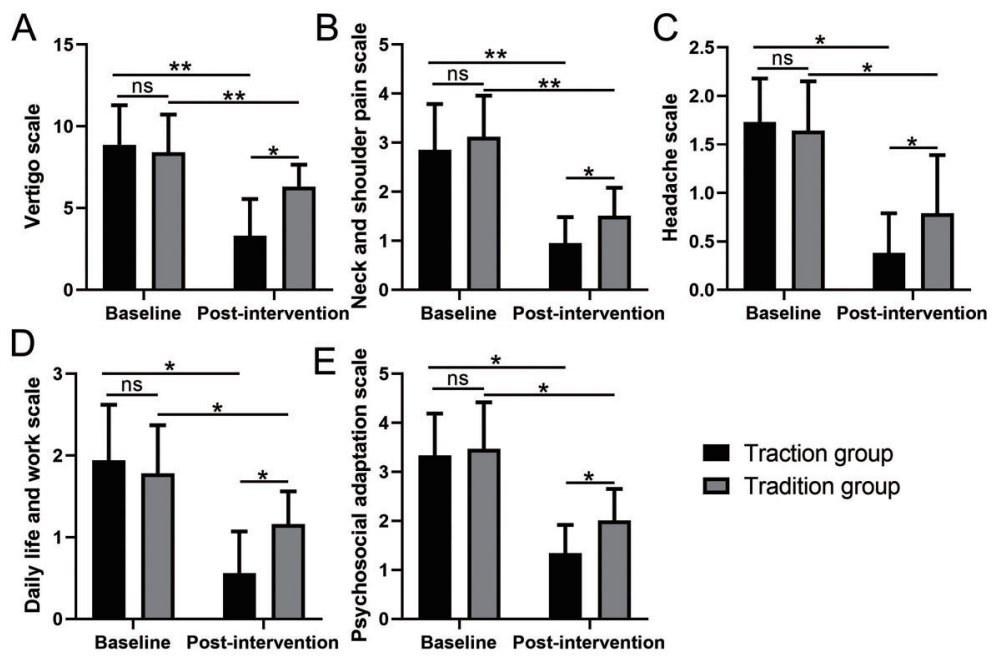

Figure 4. Effects of traction therapy on cervical vertigo symptoms and function (A-E) of patients with cervical vertigo due to atlantoaxial joint dislocation. Data are reported as means $\pm \mathrm{SD}$. ${ }^{*} \mathrm{P}<0.05$, ${ }^{* *} \mathrm{P}<0.01$, paired $t$-test and unpaired $t$-test. ns: not significant. 
Table 3. Assessment of quality of life before and after the interventions.

\begin{tabular}{|c|c|c|c|}
\hline \multirow[t]{2}{*}{ MOS-SF36 (0-100) } & \multicolumn{2}{|c|}{ Study group } & \multirow[t]{2}{*}{$P$ value } \\
\hline & Traction group $(n=42)$ & Tradition group $(n=40)$ & \\
\hline \multicolumn{4}{|l|}{ Physical function } \\
\hline Baseline & $79.12 \pm 12.14$ & $81.21 \pm 12.37$ & 0.226 \\
\hline Post-intervention & $93.24 \pm 17.5$ & $87.33 \pm 16.82$ & 0.044 \\
\hline$P$ value & 0.024 & 0.039 & \\
\hline \multicolumn{4}{|l|}{ Role function } \\
\hline Baseline & $67.65 \pm 23.18$ & $66.15 \pm 19.79$ & 0.241 \\
\hline Post-intervention & $78.23 \pm 18.19$ & $70.65 \pm 19.93$ & 0.157 \\
\hline$P$ value & 0.182 & 0.315 & \\
\hline \multicolumn{4}{|l|}{ Physical pain } \\
\hline Baseline & $56.7 \pm 17.13$ & $57.49 \pm 19.21$ & 0.562 \\
\hline Post-intervention & $80.35 \pm 19.62$ & $70.21 \pm 16.57$ & 0.043 \\
\hline$P$ value & 0.029 & 0.041 & \\
\hline \multicolumn{4}{|c|}{ General health perception } \\
\hline Baseline & $57.45 \pm 18.36$ & $56.88 \pm 19.97$ & 0.356 \\
\hline Post-intervention & $75.21 \pm 17.81$ & $63.55 \pm 16.81$ & 0.076 \\
\hline$P$ value & 0.031 & 0.084 & \\
\hline \multicolumn{4}{|l|}{ Social function } \\
\hline Baseline & $71.12 \pm 17.08$ & $72.33 \pm 19.05$ & 0.624 \\
\hline Post-intervention & $87.36 \pm 18.19$ & $79.58 \pm 19.62$ & 0.069 \\
\hline$P$ value & 0.027 & 0.135 & \\
\hline \multicolumn{4}{|l|}{ Mental function } \\
\hline Baseline & $66.38 \pm 19.16$ & $65.12 \pm 18.63$ & 0.437 \\
\hline Post-intervention & $71.06 \pm 20.13$ & $73.05 \pm 19.07$ & 0.377 \\
\hline $\mathrm{P}$ value & 0.172 & 0.243 & \\
\hline
\end{tabular}

Data are reported as means $\pm S D$. $P$ values were derived from paired $t$-test or Wilcoxon signed rank test, as appropriate between baseline vs post-intervention. $P$ values were derived from unpaired $t$-test or Mann-Whitney test, as appropriate between groups. Bold type indicates statistically significant.

The principle of manual therapy is to restore the mechanical balance of the cervical spine and, thus, relieve vertigo. At present, manual therapy is widely used in clinics since it is easy to perform, inexpensive, and relatively safe. In this study, the benefit of traction therapy was investigated. We showed that, overall, the clinical efficacy of traction therapy was higher than that of the control group. Traction therapy also led to significantly greater improvements of cervical vertigo symptoms and function and the quality of life than the traditional therapy. The LADS, which pathoanatomically relates to the severity of AJD, also decreased, confirming that traction therapy led to a significantly higher alleviation of disease progression.

The benefits of traction therapy are attributed to an increased blood flow and reduced pain associated with the procedure $(15,16)$. Traction therapy, which increases disk height, could result in lower internal pressure and decreased irritation of pain-sensitive fibers. The tensile stress also stretches the intervertebral disk and pulls the spine apart, and the muscles relax under the tension, making it easier to induce movement of the spinal process. In contrast, the traditional therapy to rotate the spinal process allows relatively less controllability and caries a higher risk of injuries of tissues, blood vessels, and nerves. The theoretical basis underlying the benefit of traction therapy in reducing cervical vertigo needs further investigation.

Our study is limited by the relatively small number of subjects. Also, we did not investigate the duration of the effects of therapy and long-term therapy was not implemented. We did not track the subjects' daily activities and, therefore, we cannot rule out the influence of other activities, such as exercises, on the conclusions. In addition, it is worth investigating whether our conclusions still hold when a mixed regression model is used for statistical analysis, a method considered more suitable for continuous data in randomized controlled trials and which lowers error of multiplicity. Further studies that address these limitations are warranted.

\section{Conclusion}

This randomized controlled trial confirmed that traction therapy was superior to traditional therapy in terms of 
clinical efficacy, reduction of LADS, and improvement of vertigo symptoms and quality of life. The results of the study supported the clinical application of traction therapy in AJD to achieve a better outcome.

\section{References}

1. Weisskopf M, Naeve D, Ruf M, Harms J, Jeszensky D. Therapeutic options and results following fixed atlantoaxial rotatory dislocations. Eur Spine J 2005; 14: 61-68, doi: 10.1007/s00586-004-0772-7.

2. Goel A, Sharma P. Craniovertebral realignment for basilar invagination and atlantoaxial dislocation secondary to rheumatoid arthritis. Neurol India 2004; 52: 338-341.

3. Yang SY, Boniello AJ, Poorman CE, Chang AL, Wang S, Passias PG. A review of the diagnosis and treatment of atlantoaxial dislocations. Global Spine J 2014; 4: 197-210, doi: 10.1055/s-0034-1376371.

4. Reid SA, Rivett DA. Manual therapy treatment of cervicogenic dizziness: a systematic review. Man Ther 2005; 10: 4-13, doi: 10.1016/j.math.2004.03.006.

5. Moser N, Mior S, Noseworthy M, Côté P, Wells G, Behr M, et al. Effect of cervical manipulation on vertebral artery and cerebral haemodynamics in patients with chronic neck pain: a crossover randomised controlled trial. $B m j$ Open 2019; 9: e0252019, doi: 10.1136/bmjopen-2018-02 5219.

6. Bracher ES, Almeida $\mathrm{Cl}$, Duprat AC, Bracher CB. A combined approach for the treatment of cervical vertigo. $J$ Manipulative Physiol Therap 2000; 23: 96-100, doi: 10.1016/S0161-4754(00)90074-5.

7. Heikkilä $H$, Johansson M, Wenngren BI. Effects of acupuncture, cervical manipulation and NSAID therapy on dizziness and impaired head repositioning of suspected cervical origin: a pilot study. Man Ther 2000; 5: 151-157, doi: $10.1054 /$ math.2000.0357.

8. Papa L, Amodio A, Biffi F, Mandara A. Impact of osteopathic therapy on proprioceptive balance and quality of life in patients with dizziness. J Bodyw Mov Ther 2017; 21: 866-872, doi: 10.1016/j.jbmt.2017.03.001.

9. F Wu, SC Bai. Effect of triple therapy on cervical vertigo caused by atlantoaxial joint disorder [in Chinese]. Shandong J Trad Chin Med 2009; 28: 39-41, <http://www.sdzy.cbpt. cnki.net/WKC/WebPublication/paperDigest.aspx?paperID= 3963787e-1340-4964-9fcd-9c4090845a15>.

10. Wang K, Wang H, Deng Z, Li Z, Zhan H, Niu W, et al. Cervical traction therapy with and without neck support: A finite element analysis. Musculoskelet Sci Pract 2017; 28: 1-9, doi: 10.1016/j.msksp.2017.01.005.

11. Wong LKF, Luo Z, Kurusu N. The effect of traction position in cervical traction therapy based on dynamic simulation

\section{Acknowledgments}

The study was supported by the Project of Natural Science Foundation of Fujian Province (2017J0105).

models. J Biomed Sci Engin 2017; 10: 243-256, doi: 10.4236/jbise.2017.105019.

12. Liu YQ, Yu HQ, Gao ZL. Effect of cervical traction at the same angle on cervical vertigo [in Chinese]. Chin J Phys Med Rehab 2005; 7: 431, <http://www.cqvip.com/QK/ 95475A/20057/16140201.html > .

13. Li YW. Treatment of cervical vertigo by massage and foot massage in traction state [in Chinese]. Modern Rehab 2001; 4: 122-123, <http://www.cqvip.com/QK/80481X/200104/511 6144.html $>$.

14. Shen M. An analysis of treating 128 cases of cervical vertigo by massage plus cervical traction [in Chinese]. Clin J Chin Med 2017; 17: 41-42, doi: 10.3969/j.issn.1674-7860.2017. 17.021 .

15. Grover S, Khanna GL. To study vertebral artery blood flow velocity during cervical c1-c2 manual traction-mulligan. J Exerc Sci Physiother 2017; 13: 23-30, doi: 10.18376/ jesp/2017/v13/i1/111269.

16. Gŭlşen M, Atici E, Aytar A, Sahin FN, et al. Effects of traction therapy in addition to conventional physiotherapy modalities on pain and functionality in patients with lumbar disc herniation: randomized controlled study. Acta Medica 2018; 34: 2017.

17. Thackeray A, Fritz JM, Childs JD, Brennan GP. The effectiveness of mechanical traction among subgroups of patients with low back pain and leg pain: a randomized trial. J Orthop Sports Phys Ther 2016; 46: 144-154, doi: 10.2519/ jospt.2016.6238.

18. Qayyum S, Waqas S, Asim HM. Outcomes of mechanical traction and manual therapy in c5-c6 cervical spondylosis for radicular pain relief. Pakistan J Med Health Sci 2017; 11: 1100-1102.

19. Chuhuai W, Dahong Z. A preliminary evaluation of symptoms and functions of patients with cervical vertigo [in Chinese]. Chin J Rehab Med 1998; 6.

20. Meijer CJ, Schene AH, Koeter MWJ. Quality of life in schizophrenia measured by the MOS SF-36 and the Lancashire quality of life profile: a comparison. Acta Psychiatr Scand 2002; 105: 293-300, doi: 10.1034/j.16000447.2002.1198.x.

21. Goel A, Figueiredo A, Maheshwari S, Shah A. Atlantoaxial manual realignment in a patient with traumatic atlantoaxial joint disruption. J Clin Neurosci 2010; 17: 672-673, doi: 10.1016/j.jocn.2009.09.023. 